\title{
Trends of LSD1 inhibitors in viral infections
}

\author{
Clemens Zwergel ${ }^{1}$, Giulia Stazi ${ }^{1}$, Antonello Mai ${ }^{1,2}$ \& Sergio Valente*,1 \\ ${ }^{1}$ Department of Drug Chemistry \& Technologies, Sapienza University of Rome, Piazzale Aldo Moro 5, 00185 Rome, Italy \\ ${ }^{2}$ Pasteur Institute - Fondazione Cenci Bolognetti, Sapienza University of Rome, Piazzale Aldo Moro 5, 00185, Rome, Italy \\ *Author for correspondence: sergio.valente@uniroma1.it
}

First draft submitted: 1 March 2018; Accepted for publication: 3 March 2018; Published online: 22 May 2018

Keywords: chromatin $\bullet$ epigenetics $\bullet$ gene expression $\bullet$ histone lysine demethylase $1 \bullet$ histone lysine demethylase 1 inhibitors $\bullet$ latency $\bullet$ viral infection

LSD1 was described as an FAD-dependent MAO homolog by Shi et al. in 2004 as the first histone demethylase [1], confirming that histone methylation is a reversible epigenetic mark. LSD1 possesses three main domains containing the N-terminal SWIRM domain, the C-terminal AOL catalytic domain and a central tower domain that contains the binding sites for the following interacting proteins: CoREST, CtBP1, HDAC1/2 and Snail1 [2]. LSD1 is capable of demethylating mono- and dimethyl H3K4 and H3K9 [1,2], although more recently it has been shown that the neuron-specific isoform LSD1n has a different substrate, histone H4K20 [3].

Moreover, several nonhistone proteins, such as p53, E2F1, DNMT and MYPT1, have been reported as LSD1 substrates [4]. LSD1 can also interact with several transcription factors that modulate LSD1 involvement in different biological contexts [4]. LSD1 is currently studied mainly in cancer, but due to its wide biological role, its dysfunction is more and more investigated also in neurodegenerative diseases, protein conformation disorders, inflammation, adipogenesis, muscle differentiation, cardiovascular diseases and in viral infections and latency [4].

DNA viruses are encapsulated without histones, but rapidly acquire chromatin structure upon infection [5], making it feasible that a histone methyltransferase or demethylase could be involved in the regulation of DNA virus replication. In the last years, a number of studies confirmed that inhibition of LSD1 can block viral genome transcription and replication of DNA viruses. In contrast, RNA viruses do not rely on chromatin structure and histone capsid for their replication. As a result, LSD1 is believed to possess little influence on RNA virus replication via histone demethylation. Nevertheless, it cannot be ruled out that LSD1 downregulates RNA virus replication via the demethylation of other host or viral proteins [5].

\section{Initial studies}

In 2010, Liang et al. demonstrated that after the infection of HSV or VZV, LSD1 was recruited to the viral IE promotors via the cellular transcriptional coactivator HCF-1 in order to enable the expression of viral IE genes. Downregulation or inhibition of LSD1 led to the accumulation of repressive chromatin, thus resulting in the inhibition of viral cycle. The reactivation of HSV from latency in sensory neurons could be hindered by using LSD1i, confirming the crucial role of this demethylase in viral infection and reactivation [6]. Together with HDAC1, -2 and CoREST/REST (HLCR), LSD1 forms a multiprotein complex that, during the HSV infection, enhancing the expression of early genes while repressing the later ones [7], leads to viral genome silencing as well as latency establishment [8].

Disruption of HLCR complex in HSV latently infected cells not only interrupts latency, but also increases HSV virulence [9]. As the HLCR is also part of the host innate immunity, it is quite evident that in HSV infection this complex is on the one hand required and on the other detrimental $[8,10]$.

One year later, the LSD1/CoREST complex was linked to the HIV promoter in vivo, through K51 demethylation and activation of Tat protein. Upon LSD1 inhibition by phenelzine, an unspecific MAO/LSD1i, HIV activation was suppressed in latently infected T cells. Hence, LSD1/CoREST normally known as transcriptional repressor is also capable to activate HIV transcription [11]. 


\section{Recent work}

Alarcon et al. demonstrated that by inhibiting LSD1 demethylase with pargyline, an irreversible nonselective MAOi, hepatitis B gene expression was repressed. cccDNA inside the hepatocyte nucleus is important for the development of HBV progeny. The repressive state of cccDNA is promoted by LSD1, so inhibition or inactivation of LSD1 leads to transcriptionally increased $\mathrm{H} 3 \mathrm{~K} 4$ methylation on viral promoters, such as viral transactivator protein $\mathrm{HBx}$, thus repressing viral gene expression. The authors have chosen pargyline for the treatment of HBV chronically infected patients, despite being a nonspecific LSD1 inhibitor. Nevertheless, their results shed light on the mechanisms of HBV regulation, potentially leading to a more efficient HBV treatment [12].

Liu et al. in their recent work highlighted that LSD1 might be an attractive therapeutic target for the treatment of cancer caused by HPV infection. LSD1 can bind to HPV16 E7, a regulator of HPV transcription, being responsible for the HPV16 E7-induced EMT and tumor metastasis in cervical cancer. LSD1 knockdown provided the opposite effect and attenuated the HPV16 E7-induced EMT [13]. So far, there are good evidences suggesting LSD1 as a potential target in viral-mediated cancers. However, further investigations are required to support the development of novel therapeutic strategies in this field.

Shan et al. demonstrated that the LSD1 inhibitor TCP strongly inhibited the IFITM3-mediated antiviral activity by increasing its K88 methylation in an influenza A virus (IAV) mouse model. TCP administration led to worsening pathology, as confirmed by severe bodyweight loss and increased mortality. Thus, these results underline that methylation of IFITM3 might be crucial in disease development in IAV-infected rodents. Hence, the induction of LSD1 expression and upregulation could be an alternative strategy for anti-flu therapy. On the other hand, as LSD1 inhibition hampers IFITM3-mediated antiviral activity, it could be interesting to apply this strategy to increase RNA virus propagation in order to assist vaccine development or infection models [5].

\section{Patent literature}

In the last years, several interesting patents regarding the involvement of LSD1 in viral infections were also published: Oryzon Genomics described TCP and TCP acetamide derivatives as LSD1 selective or LSD1/MAO-B dual inhibitors for the treatment and prevention of viral infections caused by HSV, adenovirus, HPV, parvovirus B19, smallpox virus, vaccinia virus, hepadnaviridae, polyoma virus, JC virus, for treating and preventing virus reactivation after latency, and for treating HIV in an individual coinfected with HSV2. Furthermore, these compounds have been evaluated also against the HSV-1 and -2, VZV, Epstein-Barr virus (EBV), HBV, adenovirus E1A and $\alpha$-herpes virus reactivation [14]. In more detail, they studied the case of infections caused by Flaviviridae, including HCV [6]. Effective LSD1i have been identified via a novel anti-HCV assay by using an HCV RNA replicon with luciferase reporter in the Huh7 ET cell line [14].

\section{Conclusion \& future perspective}

Almost 15 years have passed since the discovery of LSD1, and many researchers assessed its contribution in carcinogenesis and cancer progression; however there is growing evidence in scientific articles and patent literature that LSD1 plays a crucial role in viral processes such as latency, infections and viral protein expression. Viral infections are a hot topic that needs to be further investigated as often there are no sufficient therapy options. The virus takes advantage of most of the host epigenetic machinery to initiate and control its replication, hence the idea to block the viral replication and spread through administering epigenetic modulators, such as LSD1 inhibitors, might be a feasible therapeutic strategy which needs deeper evaluation. As an additional desired side effect, LSD1 inhibitors might be also useful tools to revert viral latency, helping to eradicate resistant infections. Specific LSD1 inhibitors to be studied in the viral context are already available thanks to the screenings aimed to the discovery of LSD1i for cancer treatment. To date, mainly irreversible TCP-based LSD1i have been described and entered the clinical arena [15].

Looking at future clinical applications, this might be a disadvantage as they might potentially lead to side effects, thus a careful study of the pharmacological and toxicological profile is required. However, there are already different reversible LSD1i reported in the literature, but they possess just a modest potency in vivo.

Therefore, deeper studies are necessary to firmly prove the implication of LSD1 in viral processes leading to a better understanding of the biochemical pathways behind. Furthermore, novel reversible LSD1 modulators need to be designed and the already known inhibitors optimized for improving their specificity, potency and pharmacokinetic properties [14]. 
The ternary CoREST/LSD1/HDAC1 complex has attracted special interest in cancer [16,17]. In this regard, very recently Kalin et al. designed and synthesized a first-in-class hybrid compound able to simultaneously target HDAC1 and LSD1 (corin). This dual-acting compound, when tested against a panel of melanoma and cutaneous squamous carcinoma cell lines, displayed higher antiproliferative activity with respect to single-target inhibitors alone or in combination [18]. As HDAC inhibitors are validated in various viral infections and latency [19], it would be of interest to investigate the combination of HDACi and LSD1i as well as dual HDAC/LSD1 inhibitors in this field. The rather modern approach of dual targeting could be also applied to the classical antiviral drugs and LSD1 inhibitors, either as drug combination or as novel dual-acting hybrid drugs.

Overall, LSD1 represents an emerging target to be addressed in antiviral therapy. We could expect in the years to come novel innovative therapies to fight infections, which are difficult to cure with today's drug arsenal.

\section{Financial \& competing interests disclosure}

The authors have no relevant affiliations or financial involvement with any organization or entity with a financial interest in or financial conflict with the subject matter or materials discussed in the manuscript. This includes employment, consultancies, honoraria, stock ownership or options, expert testimony, grants or patents received or pending, or royalties.

No writing assistance was utilized in the production of this manuscript.

\section{References}

1. Shi Y, Lan F, Matson C et al. Histone demethylation mediated by the nuclear amine oxidase homolog LSD1. Cell 119(7), 941-953 (2004).

2. Chen Y, Yang Y, Wang F et al. Crystal structure of human histone lysine-specific demethylase 1 (LSD1). Proc. Natl Acad. Sci. USA 103(38), 13956-13961 (2006).

3. Wang J, Telese F, Tan Y et al. LSD1n is an H4K20 demethylase regulating memory formation via transcriptional elongation control. Nat. Neurosci. 18(9), 1256-1264 (2015).

4. Zheng YC, Ma J, Wang Z et al. A systematic review of histone lysine-specific demethylase 1 and its inhibitors. Med. Res. Rev. 35(5), 1032-1071 (2015).

5. Shan J, Zhao B, Shan Z et al. Histone demethylase LSD1 restricts influenza A virus infection by erasing IFITM3-K88 monomethylation. PLoS Pathog. 13(12), e1006773 (2017).

6. Liang Y, Vogel JL, Narayanan A, Peng H, Kristie TM. Inhibition of the histone demethylase LSD1 blocks alpha-herpesvirus lytic replication and reactivation from latency. Nat. Med. 15(11), 1312-1317 (2009).

7. Gu H, Roizman B. Engagement of the lysine-specific demethylase/HDAC1/CoREST/REST complex by herpes simplex virus 1 . J. Virol. 83(9), 4376-4385 (2009).

8. Zhou G, Du T, Roizman B. The role of the CoREST/REST repressor complex in herpes simplex virus 1 productive infection and in latency. Viruses 5(5), 1208-1218 (2013).

9. Du T, Zhou G, Khan S, Gu H, Roizman B. Disruption of HDAC/CoREST/REST repressor by dnREST reduces genome silencing and increases virulence of herpes simplex virus. Proc. Natl Acad. Sci. USA 107(36), 15904-15909 (2010).

10. Zhou G, Te D, Roizman B. The CoREST/REST repressor is both necessary and inimical for expression of herpes simplex virus genes. MBio 2(1), e00313-e00310 (2011).

11. Sakane N, Kwon HS, Pagans $S$ et al. Activation of HIV transcription by the viral Tat protein requires a demethylation step mediated by lysine-specific demethylase 1 (LSD1/KDM1). PLoS Pathog. 7(8), e1002184 (2011).

12. Alarcon V, Hernandez S, Rubio L et al. The enzymes LSD1 and Set1A cooperate with the viral protein HBx to establish an active hepatitis B viral chromatin state. Sci. Rep. 6, 25901 (2016).

13. Liu Y, Wang Y, Chen C et al. LSD1 binds to HPV16 E7 and promotes the epithelial-mesenchymal transition in cervical cancer by demethylating histones at the Vimentin promoter. Oncotarget 8(7), 11329-11342 (2017).

14. Stazi G, Zwergel C, Valente S, Mai A. LSD1 inhibitors: a patent review (2010-2015). Expert Opin. Ther. Pat. 26(5), 565-580 (2016).

15. Hosseini A, Minucci S. A comprehensive review of lysine-specific demethylase 1 and its roles in cancer. Epigenomics 9(8), 1123-1142 (2017).

16. Lee MG, Wynder C, Bochar DA, Hakimi MA, Cooch N, Shiekhattar R. Functional interplay between histone demethylase and deacetylase enzymes. Mol. Cell Biol. 26(17), 6395-6402 (2006).

17. Forneris F, Battaglioli E, Mattevi A, Binda C. New roles of flavoproteins in molecular cell biology: histone demethylase LSD1 and chromatin. FEBS J. 276(16), 4304-4312 (2009).

18. Kalin JH, Wu M, Gomez AV et al. Targeting the CoREST complex with dual histone deacetylase and demethylase inhibitors. Nat. Commun. 9(1), 53 (2018). 
19. Zwergel C, Valente S, Jacob C, Mai A. Emerging approaches for histone deacetylase inhibitor drug discovery. Expert Opin. Drug Discov. 10(6), 599-613 (2015). 